\title{
Cold Agglutinin Disease: A Flash, Bedside Card, Diagnosis
}

\author{
Matteo Coen*1,2, Audrey Poirot ${ }^{3}$ and Jacques Serratrice ${ }^{1}$ \\ ${ }^{1}$ Department of Internal Medicine, Rehabilitation, and Geriatrics, Service of Internal Medicine, Geneva University Hospitals, Switzerland \\ ${ }^{2}$ Department of Pathology and Immunology, University of Geneva, Switzerland \\ ${ }^{3}$ Department of Internal Medical Specialties, Service of Oncology, Geneva University Hospitals, Switzerland
}

Received: April 16, 2018; Published: April 25, 2018

*Corresponding author: Matteo Coen, Service of Internal Medicine, Department of Internal Medicine Rehabilitation and Geriatrics, Department of Pathology and Immunology, Geneva University Hospitals, Geneva, Switzerland, Geneva University Hospitals, rue Gabrielle Perret-Gentil 4, 1211, Geneva, Switzerland, Email: matteo.coen@hcuge.ch

\section{Introduction}

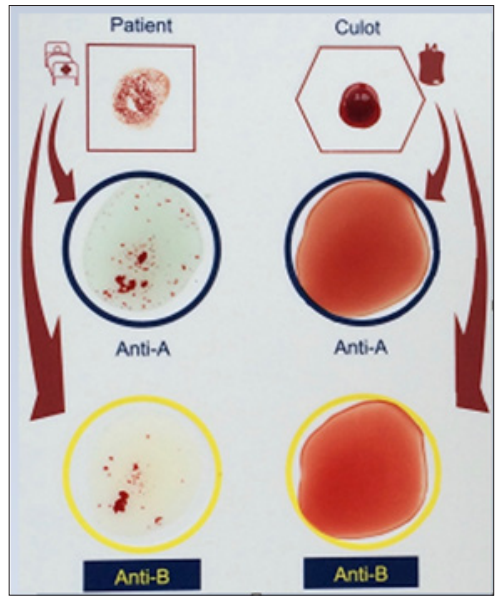

Figure 1: Blood grouping card showed agglutination of patient's blood (right, "patient") with anti-A and anti-B antibodies and in the control well, while donor's blood (left, "culot") showed no reaction.

An 89-year-old man was admitted to our service for worsening asthenia and dyspnea over a period of 3 weeks. His history was positive for arterial hypertension, ischemic heart disease, and lowgrade non-Hodgkin's lymphoma (lymphoplasmacytic lymphoma) treated with chemotherapy consisting of rituximab, prednisone, doxorubicin, cyclophosphamide and vincristine (R-CHOP). Moreover, he suffered multiple episodes of cold agglutinin anaemia secondary to lymphoma. Routine laboratory showed mild anaemia (haemoglobin of $7.6 \mathrm{~g} / \mathrm{dl}$ ); the remainder of his laboratory evaluation was within normal limits apart from mild deficiency of folic acid and vitamin B-12. Because of his cardiac condition transfusion threshold was set at $8.0 \mathrm{~g} / \mathrm{dl}$ and blood transfusion was ordered. Patient's group was $\mathrm{O} \mathrm{Rh}+$. The hospital blood transfusion laboratory detected the presence of cold agglutinins. The nurse routinely verified recipient's and donor's group at the patient's bedside prior to transfusion. The bedside blood grouping card showed the agglutination of the patient's blood with both anti-A and anti-B antibodies as well as in the control well. Instead, the donor's blood ("culot") showed no reaction (Figure 1). Transfusion was performed using a blood warmer and proved uneventful. Yet, the peculiar results of blood grouping card drove our curiosity and prompted us to review the pathophysiology of cold agglutinin haemolytic anaemia. After transfusion, patient's conditions rapidly ameliorated and was discharged at day 7 .

\section{Discussion}

The peculiar hemagglutination pattern (polyagglutination, i.e. clumping in the wells coated with anti-A and anti-B antibodies, as well as in the uncoated control well) of a $\mathrm{O} \mathrm{Rh}+$ blood at room temperature is consistent with the presence of cold agglutinins in the patient's blood. Cold agglutinins are autoantibodies (most often IgM with $\kappa$ light chains; rarely: IgA or IgG) capable of binding to polysaccharide antigens (I antigen) at the erythrocyte membrane thus provoking agglutination and complement-mediated haemolysis at low temperatures (below $37^{\circ} \mathrm{C}$ ) [1] It should be noted that unharmful, physiologic cold agglutinins are normally found; compared to their pathologic counterparts they occur at low titers $(<1: 64$ versus $>1: 512)$ and have lower thermal level of reactivity (i.e they are inactive at $>15^{\circ}$ ) [2].

Cold agglutinin disease (CAD) is a rare form of autoimmune haemolytic anaemia. CAD can be primary, or idiopathic, without overt underlying disease, or secondary, associated to hematologic malignancies, some infections (e.g. Mycoplasma pneumoniae, EBV, HIV) and -albeit rarely- some adenocarcinomas [3]. Interestingly, the most common underlying malignancy seems to be the lymphoplasmacytic lymphoma, as in the case of our patient [4]. Besides anaemia-related symptoms, patients with CAD can experience haemoglobinuria or cold-induced circulatory symptoms like acrocyanosis, Raynaud's disease and livedo reticularis 
$[3,5]$. Diagnosis of CAD relies on the assessment of anaemia and haemolysis, followed by polyspecific and monospecific direct antiglobulin test (Coombs test, to detect antibodies to the patient's erythrocytes) and measure of cold agglutinin titres [6]. The mainstay of CAD treatment is non-pharmacologic: prevention of hemolytic crisis (warm clothing, cold avoidance) and correction of anaemia (blood transfusions with an in-line blood warmer to minimize binding of agglutinins to transfused red blood cells). While treatment of secondary CAD relies on the successful treatment of the underlying disease, rituximab (in monotherapy or combined with fludarabine) can be used for primary CAD. In contrast to other autoimmune disorders, corticosteroids are useless [7].

\section{References}

1. Silberstein LE, Berkman EM, Schreiber AD (1987) Cold hemagglutinin disease associated with IgG cold-reactive antibody. Ann Intern Med 106(2): 238.

2. Pirofsky B (1985) Autoimmune Hemolytic Anemia. In Rose, NR Mackay,
IR (Eds.)., The Autoimmune Diseases, Academic Press, Orlando, Florida, pp. 485.

3. Swiecicki PL, Hegerova LT, Gertz MA (2013) Cold agglutinin disease. Blood 122(7): 1114-1121.

4. Berentsen S, Ulvestad E, Langholm R, Beiske K, Hjorth-Hansen H, et al. (2006) Primary chronic cold agglutinin disease: a population based clinical study of 86 patients. Haematologica 91(4): 460-466.

5. Berentsen S (2015) Role of Complement in Autoimmune Hemolytic Anemia. Transfus Med Hemother 42(5): 303-310.

6. Berentsen S, Tjønnfjord GE (2012) Diagnosis and treatment of cold agglutinin mediated autoimmune hemolytic anemia. Blood Rev 26(3):107-115.

7. Berentsen S (2011) How I manage cold agglutinin disease. Br J Haematol 153(3): 309-317.
This work is licensed under Creative Commons Attribution 4.0 License

Submission Link: https://biomedres.us/submit-manuscript.php

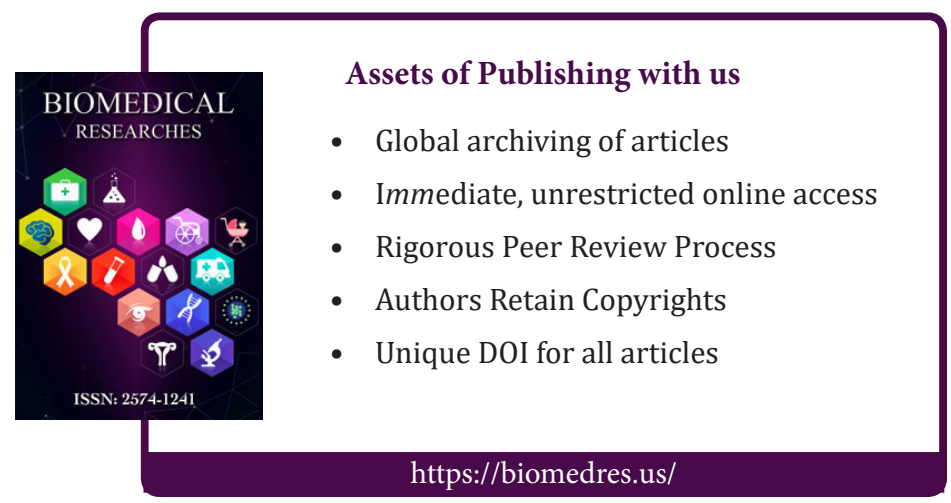

